\title{
INDEX TO VOLUME 8
}

Adler, Mark. Some finite dimensional integrable systems, 237.

Airault, Helene. On the solution of a functional equation, 245.

Baggett, Larry. A characterization of "Heisenberg groups"; when is a particle free?, 561.

Bullough, R. K., and Caudrey, P. J. The double-sine-Gordon equation: wobbling solitons?, 53.

Burbea, Jacob. On the Hessian of the Carathéodory metric, 555.

Campbell, David K. Exact classical solutions to the two-dimensional "sigma" model; an unusual application of inverse scattering techniques, 371 .

Cannon, J. W. $\Sigma^{2} H^{3}=S^{5} / G, 527$.

Carlitz, L. Polynomial characteristic functions for $G F(p)$ and irregular primes, 583.

Caudrey, P. J. See Bullough, R. K.

Corones, James. See Stewart, Richard W.

Costabile, G. See Parmentier, R. D.

Dauns, John. Enveloping W*-algebras, 589.

Driscoll, C. F., and O'Neil, T. M. Those ubiquitous, but oft unstable, lattice solitons, 211.

Faber, V. Large abelian subgroups of some infinite groups, II, 481.

Ferguson, Warren E. See Lake, Bruce M.

Flaschka, H., and McLaughlin, D. W. Preface, 1.

Flaschka, H., and McLaughlin, D. W. Introduction to mathematical techniques for continuous problems, 3.

Flaschka, H., and McLaughlin, D. W. Introduction to discrete systems, 125.

Flaschka, H., and McLaughlin, D. W. Introduction to waves in plasmas, 253.

Flaschka, H., and McLaughlin, D. W. Introduction to quantization and quantum field theory, 333.

Friedberg, Michael. On the universal compactification of a cone, 503.

Hasegawa, Akira. See Mima, Kunioki.

Hasslacher, Brosl, and Neveu, André. Non-linear quantum field theory, 341.

Helton, Jon C. Solution of two Volterra integral equations, 547.

Hinton, Don B. Deficiency orders of odd-order differential operators, 627.

Hyman, J. M. Time evolution of almost periodic solutions of the KdV equation, 95.

Ichikawa, Yoshi-hiko. See Wadati, Miki.

Jackson, E. Atlee. Nonlinearity and irreversibility in lattice dynamics, 127.

Kaigh, W. D. An elementary derivation of the distribution of the maxima of Brownian meander and Brownian excursion, 641.

Kaup, D. J. Applications of the inverse scattering transform I: self-induced transparency, 71.

Kaup, D. J. Applications of the inverse scattering transform II: the three-wave resonant interaction, 283.

Kirkman, Ellen E. The Pierce representation of an inertial coefficient ring, 533.

Kohn, Peter. Finite projective dimension under change of rings, 647.

Konno, Kimiaki. See Wadati, Miki.

Lake, Bruce M., Yuen, Henry C., and Ferguson, Warren E. Envelope solitons and recurrence in nonlinear deep water waves: theory and experiment, 105.

Lee, Y. C. See Morales, G. J.

Maxon, Stephen. Cylindrical and spherical solitons, 269.

Copyright (C) 1978 Rocky Mountain Mathematics Consortium 
McLaughlin, D. W. See Flaschka, H.

Mima, Kunioki, and Hasegawa, Akira. 2- and 3-dimensional localization of random phase waves, 309.

Morales, G. J., and Lee, Y. C. Soliton-like structures in plasmas, 257.

Münster, M. On A. Lax's condition of hyperbolicity, 443.

Münster, M. On hyperbolic polynomials with constant coefficients, 653.

Nelson, James, Jr. A common fixed point structure, 675.

Neveu, André. See Hasslacher, Brosl.

Newell, Alan C. The inverse scattering transform, nonlinear waves, singular perturbations and synchronized solitons, 25.

Nohl, Craig R. Quantization of nonlinear wave equations, 393.

Novikov, S. P. A method for solving the periodic problem for the KdV equation and its generalizations, 83.

O’Neil, T. M. See Driscoll, C. F.

Oshobi, E. O. Norm-decreasing isomorphisms of the trace-class algebras of $H^{*}$ algebras, 471.

Palmer, T. W. Classes of nonabelian, noncompact, locally compact groups, 683.

Park, Sehie. Fixed points of f-contractive maps, 743.

Parmentier, R. D., and Costabile, G. Fluxon propagation and DC current singularities in long Josephson junctions, 117.

Phillips, Keith. A note on local field duality, 433.

Prabhakar, T. R., and Rekha, Suman. Some results on the polynomials $L_{n}{ }^{\alpha, \beta}(x), 751$.

Rehka, Suman. See Prabhakar, T. R.

Sanuki, Heiji. See Wadati, Miki.

Scheiblich, H. E. On coproducts of rectangular bands, 539.

Segur, Harvey. Solitons as approximate descriptions of physical phenomena, 15.

Slud, Eric V. A note on exchangeable sequences of events, 439.

Stewart, Richard W., and Corones, James. The breakup of soliton like pulses on a nonlinear, nonuniform electrical lattice, 227.

Sutherland, Bill. A brief history of the quantum soliton with new results on the quantization of the Toda lattice, 413.

Toda, Morikazu. Problems in nonlinear dynamics, 197.

Turek, Richard J. An approach to completely ergodic transformations and a stacking method, 755.

Umamaheswaram, S. $\lambda(n, k)$-parameter families and associated convex functions, 491.

Umamaheswaram, S. $\lambda(n, k)$-convex functions, 759.

Wadati, Miki, Sanuki, Heiji, Konno, Kimiaki, and Ichikawa, Yoshi-hiko. Circular polarized nonlinear Alfvén waves-a new type of nonlinear evolution equation in plasma physics, 323.

Warren, R. H. Neighborhoods, bases and continuity in fuzzy topological spaces, 459.

Willett, D. Differential inequalities and the asymptotics of second order nonlinear differential equations, 447.

Yuen, Henry C. See Lake, Bruce M. 